\title{
Nephrometry scores and perioperative outcomes following robotic partial nephrectomy
}

Renato B. Corradi ${ }^{1}$, Emily A. Vertosick ${ }^{2}$, Daniel P. Nguyen ${ }^{1}$, Antoni Vilaseca ${ }^{1}$, Daniel D. Sjoberg ${ }^{2}$, Nicole Benfante ${ }^{1}$, Lucas N. Nogueira ${ }^{3}$, Massimiliano Spaliviero ${ }^{1}$, Karim A. Touijer ${ }^{1}$, Paul Russo ${ }^{1}$, Jonathan A. Coleman ${ }^{1}$

${ }^{1}$ Department of Surgery, Urology Service, Memorial Sloan Kettering Cancer Center, New York, USA; ${ }^{2}$ Department of Epidemiology and Biostatistics, Memorial Sloan Kettering Cancer Center, New York, USA; ${ }^{3}$ Departamento de Cirurgia, Serviço de Urologia, Hospital das Clínicas da UFMG, Belo Horizonte, MG, Brasil

\section{ABSTRACT}

Objectives: Based on imaging features, nephrometry scoring systems have been conceived to create a standardized and reproducible way to characterize renal tumor anatomy. However, less is known about which of these individual measures are important with regard to clinically relevant perioperative outcomes such as ischemia time (IT), estimated blood loss (EBL), length of hospital stay (LOS), and change in estimated glomerular filtration rate (eGFR) after robotic partial nephrectomy (PN). We aimed to assess the utility of the RENAL and PADUA scores, their subscales, and C-index for predicting these outcomes.

Materials and Methods: We analyzed imaging studies from 283 patients who underwent robotic PN between 2008 and 2014 to assign nephrometry scores (NS): PADUA, RENAL and C-index. Univariate linear regression was used to assess whether the NS or any of their subscales were associated with EBL or IT. Multivariable linear regression and linear regression models were created to assess LOS and eGFR.

Results: The three NS were significantly associated with EBL, IT, LOS, and eGFR at 12 months after surgery. All subscales with the exception of anterior/posterior were significantly associated with EBL and IT. Collecting system, renal rim location, renal sinus, exophytic/endophytic, and nearness to collecting system were significant predictors for LOS. Only renal rim location, renal sinus invasion and polar location were significantly associated with eGFR at 12 months.

Conclusions: Tumor size and depth are important characteristics for predicting robotic PN outcomes and thus could be used individually as a simplified way to report tumors features for research and patient counseling purposes.

\section{ARTICLE INFO}

\section{Keywords:}

Kidney Neoplasms;

Nephrectomy; Robotics

Int Braz J Urol. 2017; 43: 1075-83

Submitted for publication:

October 17, 2016

Accepted after revision:

February 09, 2017

Published as Ahead of Print:

May 16, 2017

\section{INTRODUCTION}

Partial nephrectomy (PN) is the preferred technique in the treatment of small $(<4 \mathrm{~cm})$ and mid-size $(<7 \mathrm{~cm})$ kidney masses and can entail varying degrees of technical challenges based on anatomic features of the tumor $(1,2)$. The growing interest in PN has highlighted the need for a standardized method for characterizing renal masses that provides clinically meaningful information when different approaches and techniques for PN are compared $(3,4)$.

In this context, different nephrometry scores (NS), principally based on renal imaging, 
have been proposed (5-7). Their main objective is to provide a reproducible way of characterizing anatomy and classifying renal masses with emphasis on the most surgically-relevant features. However, subscale metrics have not been validated individually and little is known about which of them are truly related to complications and outcomes after PN. Although some studies have investigated the impact of the individual components of NS on complications or other outcomes, their results should be taken cautiously because of small series $(8,9)$ and because they only compared subscales within the same NS (10-12). Furthermore, very few of them focused on robotic procedures $(13,14)$. This is important because trend analyses show that robotic PN is likely to become the most frequently performed operation for renal masses (15).

Here we assessed the utility of the RENAL and PADUA scores, their subscales, and C-index for predicting perioperative parameters and postoperative outcomes from a single institution series of robotic PN. We hypothesized that some subscales hold more value than others for predicting some of these outcomes, and that in daily practice the use of the most relevant subscales is as effective as the use of one of the complexity scores.

\section{MATERIALS AND METHODS}

\section{Patients}

After obtaining institutional review board approval, we identified 317 patients who underwent robotic PN between May 2008 and August 2014 at Memorial Sloan Kettering Cancer Center (MSKCC). Patients with benign histo$\operatorname{logy}(n=16)$ and whose imaging exams were not available $(n=18)$ were excluded, leaving 283 patients with malignant tumors for final analysis. Baseline characteristics were extracted from a prospectively maintained database and included patient age, gender, American Society of Anesthesiologists (ASA) score, and race.

\section{Surgical Procedures}

All procedures were performed by surgeons with over five years of experience in mi- nimally invasive and robotic surgery including PN. Normothermic ischemia was utilized during sharp excisional resection and surgical repair was conducted by renorrhaphy with absorbable sutures over nitrocellulose and thrombin-based procoagulant materials. Cases were performed trans or retroperitoneally according to surgeon preference and tumor location. Early unclamping and enucleo-resection techniques were not utilized. Postoperative care was managed under a standardized clinical care pathway overseen by care providers independent from the surgical team (16). Surgical complications reported within 30 days were obtained prospectively using a standardized grading system (17).

\section{Outcome measures}

\section{Pathologic data}

Kidney tumor specimens were evaluated according to standard pathology protocol. Pathologic data included tumor size and tumor stage according to the 2009 AJCC TNM classification (18).

\section{Postsurgical complications}

Postsurgical complications within 30 days were collected prospectively and graded using the modified Clavien classification system (19). Regular correspondence with patients and their physicians ensured that treatment received outside of our institution was accounted for in the database.

\section{Perioperative outcomes}

Estimated blood loss (EBL), ischemia time (IT) and length of stay (LOS) were collected from our prospectively maintained database. Data regarding IT and LOS were not available for 4 and 56 patients, respectively.

\section{Renal function outcomes}

Baseline eGFR was calculated from creatinine readings using the Chronic Kidney Disease Epidemiology Collaboration (CKD-Epi) equation taken one month prior to surgery (20). Postoperative eGFR was calculated at 12 months or from the closest measurement between 6 and 12 months following surgery. 


\section{Nephrometry scoring}

RENAL scores were assigned as described by Kutikov and Uzzo (6). The components of this score are radius (R), exophytic/endophytic (E), proximity to collecting system or sinus $(\mathrm{N})$, anterior/posterior $(\mathrm{A})$, and location relative to polar lines (L). R, E, N and L are scored from 1 to 3. Subscale A is a categorical variable defined as anterior, posterior or $\mathrm{X}$ when a designation relative to anterior/posterior is not possible.

The PADUA nephrometry score, described by Ficarra et al. (5), is similar to the RENAL nephrometry score. Longitudinal location, renal rim, renal sinus, and urinary collecting system are scored with either 1 or 2 points; exophytic rate and tumor size are scored from 1 to 3 points. Tumor size was defined as the maximum tumor diameter as described in the original articles from Ficarra et al. and Kutikov et al. $(5,6)$,

C-index measurements are described by Simmons et al. (7) and were made according to the authors instructions contained in the original article where this NS is described (7).

All NS measurements were made by the same urologic surgeon and were based on the original studies described.

\section{Statistical analyses}

Univariate linear regression was used to assess the association between C-index, RENAL, PADUA and any of the RENAL or PADUA NS subscales with EBL and IT. Multivariable linear regression models adjusted for age and ASA score were used to determine whether NS is associated with LOS. Linear regression models adjusted for preoperative eGFR were used to assess the association of the NS with eGFR at 12 months after surgery. The effects of the renal NS on oncologic outcomes were not studied because of the limited number of events. All analyses were performed using Stata 12 (StataCorp, College Station, TX).

\section{RESULTS}

Patient and tumor characteristics are described in Table-1. Renal nephrometry subscale scores are reported in Table-2.
Table 1 - Patient and tumor characteristics, $\mathrm{N}=283$. Data are reported as frequency (\%) or median (IQR).

\begin{tabular}{lc}
\hline Female & $93(33 \%)$ \\
Age & $60(51,67)$ \\
ASA score & \\
1 & $17(6 \%)$ \\
2 & $101(36 \%)$ \\
3 & $160(57 \%)$ \\
4 & $5(2 \%)$ \\
Pathologic T stage & \\
T0 & $4(1 \%)$ \\
T1 & $240(85 \%)$ \\
T2 & $8(3 \%)$ \\
T3 & $31(11 \%)$ \\
Pathologic N stage (N=277) & \\
N0 & $138(50 \%)$ \\
N1 & $1(<1 \%)$ \\
N2 & $4(1 \%)$ \\
NX & $134(48 \%)$ \\
Pathologic M stage (N=231) & \\
M0 & $212(92 \%)$ \\
M1 & $6(3 \%)$ \\
Mrade $3+$ complications within 30 days & \\
\hline
\end{tabular}

C-index, RENAL and PADUA scores were all significantly associated with EBL, IT, LOS, and eGFR at 12 months after surgery. The effect of a one-unit increase in RENAL score and a one-unit increase in PADUA score were similar for all outcomes.

With regard to subscales, tumor size, a component of both the PADUA and RENAL scores, was associated with perioperative outcomes: larger tumors resulted in significantly increased EBL and IT (Table-3). With the exception of the anterior/posterior scale, all other scales describing tumor location were significantly associated with EBL and IT, with tumors in complex locations or centrally located having increased EBL and longer IT (Table-3). 
Table 2 - RENAL and PADUA subscale scores, $\mathrm{N}=283$. Data are reported as frequency (\%).

\begin{tabular}{lc}
\hline Collecting sinus (PADUA) & \\
\hline Absent & $64(23 \%)$ \\
$\quad$ Dislocated/infiltrated & $219(77 \%)$ \\
Renal rim (PADUA) & \\
$\quad$ Lateral & $194(69 \%)$ \\
$\quad$ Medial & $89(31 \%)$ \\
Renal sinus (PADUA) & \\
$\quad$ Absent & $184(65 \%)$ \\
$\quad$ Renal sinus location & $99(35 \%)$ \\
Sinus line (PADUA) & \\
$\quad$ Entirely above/below or <50\% crossing & $162(57 \%)$ \\
$\quad$ sinus lines & \\
$\quad$ Entirely between / $\geq 50 \%$ crossing & $121(43 \%)$ \\
Nearness to collecting sinus (RENAL) & \\
$\quad \geq 7 m m$ & $45(16 \%)$ \\
4 mm-7 mm & $35(12 \%)$ \\
$\quad \leq 4$ mm & $203(72 \%)$ \\
Anterior/Posterior (RENAL) & \\
Anterior & $114(40 \%)$ \\
Posterior & $114(40 \%)$ \\
Neither & $55(19 \%)$ \\
Location relative to polar line (RENAL) & \\
Entirely above/below & $143(51 \%)$ \\
Crosses polar lines $<50 \%$ & $46(16 \%)$ \\
Entirely between / $\geq 50 \%$ crossing & $94(33 \%)$ \\
Exophytic / endophytic (PADUA/RENAL) & \\
$\quad \geq 50 \%$ exophytic & $108(38 \%)$ \\
$\quad<50 \%$ endophytic & $96(34 \%)$ \\
$\quad$ Entirely endophytic & $79(28 \%)$ \\
$\quad 4$ cm - 7cm & \\
\hline Size (PADUA/RENAL) & \\
\hline
\end{tabular}

Regarding LOS, collecting system, renal rim location, renal sinus, exophytic/endophytic, and nearness to collecting system were significant predictors (Table-4). Conversely, tumor size was not associated with LOS. Furthermore, only renal rim location, renal sinus invasion and polar loca- tion were significantly associated with eGFR at 12 months. It has to be noted that anterior/posterior location was not significantly associated with any peri-operative or postoperative outcomes, while renal rim location and renal sinus invasion, both from PADUA, were the only subscales associated with all four outcomes.

Tumor location relative to polar lines was found to significantly increase both EBL and IT, with patients with centrally located tumors having the worst outcomes. As a sensitivity analysis, we compared patients with centrally located tumors (entirely between or crossing polar lines more than $50 \%$ ) with patients whose tumors did not cross polar lines or crossed less than 50\%. Patients with centrally located tumors had an increase in EBL of $60 \mathrm{~mL}(95 \%$ confidence interval [CI] 12, 109, $\mathrm{p}=0.02)$ and increase in IT of 6.5 minutes $(95 \% \mathrm{CI}$ 3.8, 9.2, $\mathrm{p}<0.0001$ ) compared to patients without centrally located tumors. When comparing patients with centrally located tumors to all others, we found some evidence that these patients had longer LOS (0.30 days, 95\% CI-0.02, 0.62, $\mathrm{p}=0.07$ ), although this association did not reach conventional levels of statistical significance. These patients also had eGFR at 12 months that was 3.56 points lower (95\% CI-7.04, -0.08, $\mathrm{p}=0.045$ ) compared to patients with tumors that did not cross the polar line or crossed $<50 \%$.

\section{DISCUSSION}

Prior to the introduction of NS only limited and mainly quantitative information regarding surgically-relevant anatomical features of solid enhancing renal masses was available. By defining the characteristics of tumors treated with $\mathrm{PN}$, integrated anatomical systems can be used to predict the risk of surgical complications and allows comparisons among different surgeons and techniques. Given the variability in techniques and possibly outcomes inherent to surgical approaches, here we offer a comprehensive analysis on the value of all previously described NS and their subscales for robotic PN.

The RENAL and PADUA NS contain subscales related to tumor size and location within the renal parenchyma, as well as relative to portions 
Table 3 - Univariate linear regression models for the association between C-index, RENAL and PADUA total scores and subscales and peri-operative outcomes: estimated blood loss (EBL) in $\mathrm{mL}(\mathrm{N}=283)$ and ischemia time (IT) in minutes, ( $\mathrm{N}=279)$.

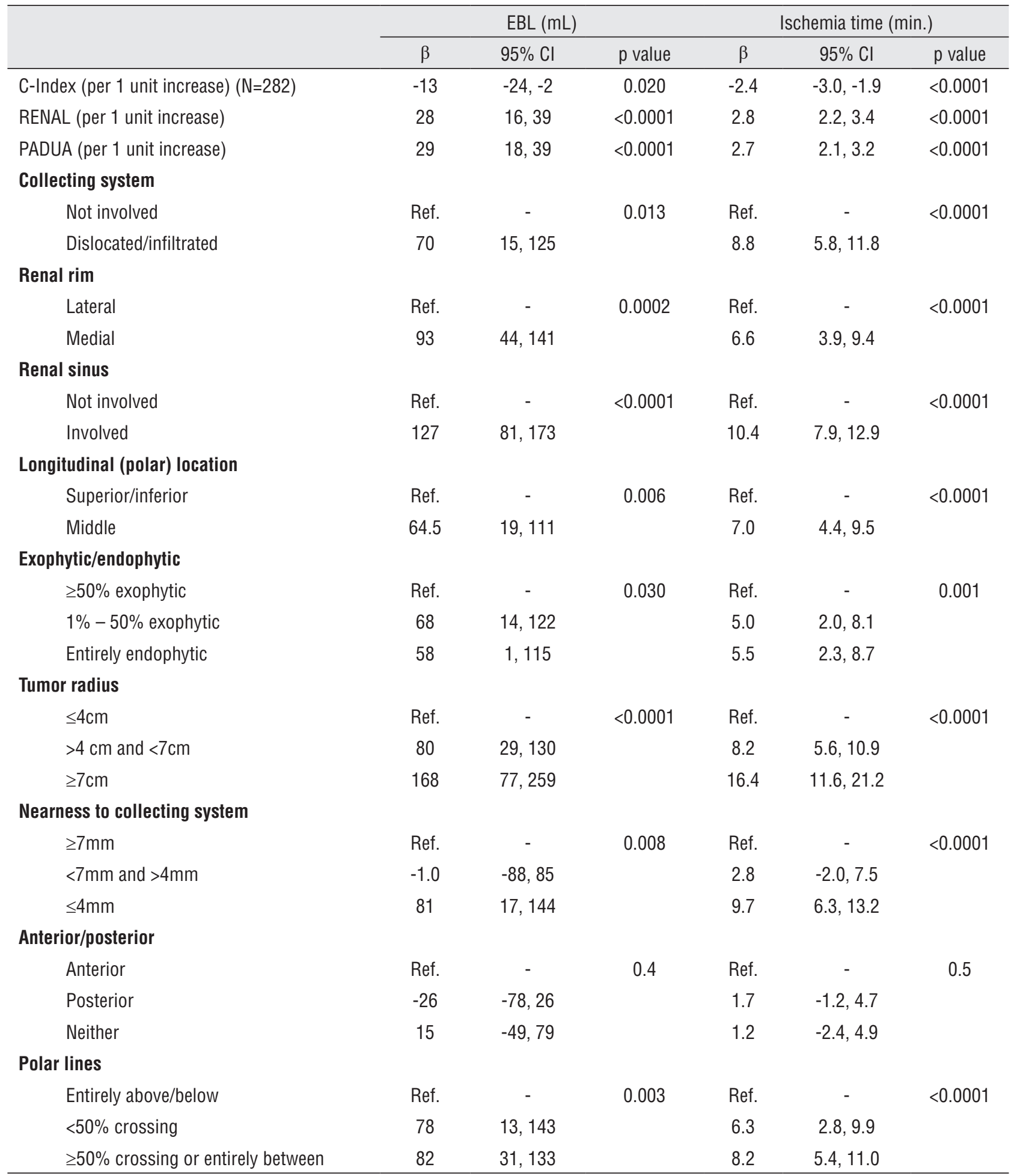

$\boldsymbol{\beta}=$ difference between patients who have that subscale score and those in the reference group

Ref. $=$ reference group 
Table 4 - Multivariable linear regression models for the association between C-index, RENAL and PADUA total scores subscales and postoperative outcomes. The model for length of stay (LOS) in days was adjusted for ASA score and age $(\mathrm{N}=227)$.

\begin{tabular}{|c|c|c|c|c|c|c|}
\hline & \multicolumn{3}{|c|}{ Length of stay } & \multicolumn{3}{|c|}{ eGFR at 12 months } \\
\hline & $\beta$ & $95 \% \mathrm{Cl}$ & $p$ value & $\beta$ & $95 \% \mathrm{Cl}$ & $p$ value \\
\hline C-Index (per 1 unit increase) $(\mathrm{N}=226)$ & -0.1 & $-0.2,-0.02$ & 0.014 & 0.95 & $0.17,1.72$ & 0.017 \\
\hline RENAL (per 1 unit increase) (N=227) & 0.14 & $0.07,0.22$ & 0.0002 & -1.05 & $-1.86,-0.23$ & 0.012 \\
\hline PADUA (per 1 unit increase) ( $N=227)$ & 0.14 & $0.07,0.21$ & 0.0002 & -1.06 & $-1.83,-0.29$ & 0.007 \\
\hline \multicolumn{7}{|l|}{ Collecting system } \\
\hline Not involved & Ref. & - & 0.001 & Ref. & - & 0.6 \\
\hline Dislocated/infiltrated & 0.6 & $0.2,1.0$ & & -1.12 & $-5.04,2.80$ & \\
\hline \multicolumn{7}{|l|}{ Renal rim } \\
\hline Lateral & Ref. & - & 0.001 & Ref. & - & 0.019 \\
\hline Medial & 0.6 & $0.3,0.9$ & & -4.24 & $-7.76,-0.72$ & \\
\hline \multicolumn{7}{|l|}{ Renal sinus } \\
\hline Not involved & Ref. & - & 0.015 & Ref. & - & 0.003 \\
\hline Involved & 0.4 & $0.1,0.7$ & & -5.17 & $-8.58,-1.77$ & \\
\hline \multicolumn{7}{|l|}{ Longitudinal (polar) location } \\
\hline Superior/inferior & Ref. & - & 0.2 & Ref. & - & 0.014 \\
\hline Middle & 0.2 & $-0.1,0.5$ & & -4.15 & $-7.47,-0.84$ & \\
\hline \multicolumn{7}{|l|}{ Exophytic/endophytic } \\
\hline$\geq 50 \%$ exophytic & Ref. & - & 0.049 & Ref. & - & 0.6 \\
\hline $1 \%-50 \%$ exophytic & 0.3 & $-0.1,0.7$ & & -1.97 & $-5.85,1.92$ & \\
\hline Entirely endophytic & 0.5 & $0.1,0.8$ & & -0.23 & $-4.35,3.89$ & \\
\hline \multicolumn{7}{|l|}{ Tumor radius } \\
\hline$\leq 4 \mathrm{~cm}$ & Ref. & - & 0.082 & Ref. & - & 0.057 \\
\hline$>4 \mathrm{~cm}$ and $<7 \mathrm{~cm}$ & 0.4 & $0.04,0.7$ & & -2.60 & $-6.27,1.06$ & \\
\hline$\geq 7 \mathrm{~cm}$ & 0.3 & $-0.3,0.9$ & & -7.26 & $-13.85,-0.68$ & \\
\hline \multicolumn{7}{|l|}{ Nearness to collecting system } \\
\hline$\geq 7 \mathrm{~mm}$ & Ref. & - & 0.002 & Ref. & - & 0.11 \\
\hline$<7 \mathrm{~mm}$ and $>4 \mathrm{~mm}$ & 0.1 & $-0.5,0.7$ & & 1.83 & $-4.40,8.07$ & \\
\hline$\leq 4 \mathrm{~mm}$ & 0.7 & $0.2,1.1$ & & -2.96 & $-7.50,1.57$ & \\
\hline \multicolumn{7}{|l|}{ Anterior/posterior } \\
\hline Anterior & Ref. & - & 0.6 & Ref. & - & 0.8 \\
\hline Posterior & -0.2 & $-0.5,0.2$ & & -0.78 & $-4.45,2.90$ & \\
\hline Neither & 0.01 & $-0.4,0.4$ & & -1.68 & $-6.25,2.89$ & \\
\hline \multicolumn{7}{|l|}{ Polar lines } \\
\hline Entirely above/below & Ref. & - & 0.2 & Ref. & - & 0.073 \\
\hline$<50 \%$ crossing & 0.3 & $-0.2,0.7$ & & -3.05 & $-7.69,1.59$ & \\
\hline$\geq 50 \%$ crossing or entirely between & 0.3 & $-0.1,0.6$ & & -4.14 & $-7.81,-0.46$ & \\
\hline \multicolumn{7}{|l|}{ Polar lines } \\
\hline Did not cross or $<50 \%$ crossing & Ref. & - & 0.069 & Ref. & - & 0.045 \\
\hline $\begin{array}{l}\geq 50 \% \text { crossing or entirely between } \\
\text { (centrally located tumors) }\end{array}$ & 0.3 & $-0.02,0.62$ & & -3.56 & $-7.04,-0.08$ & \\
\hline
\end{tabular}

The model for post-operative eGFR at 12 months was adjusted for pre-operative eGFR ( $N=279$ ).

$\boldsymbol{\beta}=$ difference in length of stay between patients with that subscale score and patients in the reference group with similar ages and ASA scores

Ref. = reference group 
of the collecting system or the renal sinus. The C-index is calculated based on the position of the tumor relative to the center of the kidney and is adjusted by tumor size. Any renal mass with a C-index $<1$ has some part superimposed on the renal center and a C-index of 1 equates to a tumor with its edge touching the middle of the kidney. Simmons and coworkers from the Cleveland Clinic (7) showed that a C-index $<2$ was associated with longer IT, higher EBL and rates of intra- and postoperative complications after laparoscopic PN. Here, concordant findings were demonstrated for robotic PN, as a one-unit increase in C-index was associated with lower EBL and shorter IT.

In the current analysis, PADUA and RENAL were associated with all outcomes studied, which is in line with previous non-robotic analyses. In a large retrospective multicenter study, Ficarra et al. showed that PADUA stratification was an independent predictor of IT longer than 20 minutes and overall complications within 3 months (12).

Smaller series also demonstrated associations between RENAL, PADUA and C-index with warm IT (4) and change in renal function $(8,21)$.

However, a main issue remains as to whether, for robotic PN, there is significant added value of using a composite index scoring system over the simple use of individual measures used to calculate the index. In our analysis that included a large series of robotic PN, all subscales from the PADUA and RENAL scores, except for anterior/ posterior, were independently associated with two important surrogates of challenging tumor resections $(1,22)$, EBL and IT. Tumor size $\geq 7 \mathrm{~cm}$ had the biggest impact on IT and EBL, being associated with IT on average 16 minutes and a mean EBL $168 \mathrm{~mL}$ higher than for tumor size $\leq 4 \mathrm{~cm}$. Concordant findings were seen in a preliminary study from our institution including 90 open, laparoscopic and robotic PN procedures in which location relative to polar line, sinus involvement, tumor size, collecting system involvement, and C-index score were all associated with increased IT (9). Along these lines, Tsivian et al. reported that tumor size, endophytic growth and both central and hilar mass location were associated with increased IT (23). It has to be stressed that other parameters such as the amount of perinephric fat can bring additional difficulty to the surgical procedure (24) and affect perioperative outcomes. Along the same line, the volume of preserved parenchyma has shown prognostic value for postoperative renal function (25). Our study aimed at evaluating subscales of NS and therefore we did not evaluate these other factors.

LOS is an important outcome when evaluating surgical procedures and an accepted surrogate for immediate post-operative convalescence features including complications (1). Moreover, shortening of LOS is one of the assumed benefits of the robotic approach. Here LOS was associated with features that evaluate tumor depth of invasion, demonstrating roughly changes per each of the subscales unit increase. All cases analyzed were performed by the same operative approach (robotic PN) using a common care pathway: the LOS outcome is likely a surrogate for immediate postoperative convalescence features including complications (1). These data are consistent with those from Simhan et al. (26). Performing a multivariable analysis on 390 patients who underwent PN, the authors found that high tumor complexity using the RENAL US was associated with the occurrence of major complications. The low rate of major postoperative complications (Table-1) in our cohort precluded comparative analyses between NS and subscales.

Renal function after PN depends on several factors, mainly pre-operative eGFR, IT and the volume of preserved kidney parenchyma $(1,25,27$, 28) It seems intuitive that the more complex the renal tumors are, the longer the vessels need to be clamped to achieve safe excision and reconstruction (29). Using multivariable analysis we showed that only three PADUA subscales and nephrometry composite scores were associated with functional loss. Moreover, although the composite indexes were associated with all outcomes studied, the magnitude of difference per unit change in index score was less than for renal rim, sinus involvement and polar location. Thus, our data question the utility of composite index scoring compared to individual subscale for robotic PN.

The ideal method to describe renal masses should not only accurately reflect surgical complexity, but also be reproducible and objective in or- 
der to be applicable in daily practice. The reduction of subscales reported in each score might augment each method's reproducibility and applicability.

Although our database is prospectively maintained, LOS missing for 56 patients due to loss of electronic data. Another limitation of our study is the retrospective nature of the analysis, which makes our results vulnerable to caveats in patient selection and other unrecognized confounding factors. However, NS scoring was done prospectively as we evaluated all previously described NS. Another strength of the study was the use of a large series of robotic PN, thus limiting variabilities related to technique compared to previous studies.

In our analysis, we show that the relevant NS features are those that describe tumor depth of invasion and size. It is interesting that tumor size was a significant predictor despite the fact that median tumor size in this study was $2.9 \mathrm{~cm}$. Intuitively, this would be expected for larger tumors only. This suggests potential for solely using subscales as a simplified way to stratify kidney tumors for case mix adjustments in programs of quality assurance, for example, when comparing the outcomes of different surgeons and techniques. Furthermore, individual subscale metrics can be a simplified way for the practicing urologist in his/her clinical practice to counsel patients about surgical risks.

\section{ACKNOWLEDGEMENTS}

The authors thank MSKCC editorial office for their review of the manuscript.

Daniel P. Nguyen is a research fellow and is supported by research grants from the Nuovo-Soldati, the Arnold U. und Susanne Huggenberger-Bischoff, the Bangerter Foundations and the Swiss Urological Association (Switzerland).

\section{CONFLICT OF INTEREST}

None declared.

\section{REFERENCES}

1. Russo P. Partial nephrectomy for renal cancer: Part I. BJU Int. 2010;105:1206-20.
2. Touijer K, Jacqmin D, Kavoussi LR, Montorsi F, Patard JJ, Rogers CG, et al. The expanding role of partial nephrectomy: a critical analysis of indications, results, and complications. Eur Urol. 2010;57:214-22.

3. Kutikov A, Smaldone MC, Uzzo RG. Partial versus radical nephrectomy: balancing nephrons and perioperative risk. Eur Urol. 2013;64:607-9.

4. Bylund JR, Gayheart D, Fleming T, Venkatesh R, Preston DM, Strup SE, Crispen PL. Association of tumor size, Iocation, R.E.N.A.L., PADUA and centrality index score with perioperative outcomes and postoperative renal function. $J$ Urol. 2012;188:1684-9.

5. Ficarra V, Novara G, Secco S, Macchi V, Porzionato A, De Caro $R$, et al. Preoperative aspects and dimensions used for an anatomical (PADUA) classification of renal tumours in patients who are candidates for nephron-sparing surgery. Eur Urol. 2009;56:786-93.

6. Kutikov A, Uzzo RG. The R.E.N.A.L. nephrometry score: a comprehensive standardized system for quantitating renal tumor size, location and depth. J Urol. 2009;182:844-53.

7. Simmons MN, Ching CB, Samplaski MK, Park CH, Gill IS. Kidney tumor location measurement using the $\mathrm{C}$ index method. J Urol. 2010;183:1708-13.

8. Okhunov Z, Rais-Bahrami S, George AK, Waingankar N, Duty B, Montag S, et al. The comparison of three renal tumor scoring systems: C-Index, P.A.D.U.A., and R.E.N.A.L. nephrometry scores. J Endourol. 2011;25:1921-4.

9. Spaliviero M, Poon BY, Aras O, Di Paolo PL, Guglielmetti GB, Coleman CZ, et al. Interobserver variability of R.E.N.A.L., PADUA, and centrality index nephrometry score systems. World J Urol. 2015;33:853-8.

10. Rosevear HM, Gellhaus PT, Lightfoot AJ, Kresowik TP, Joudi FN, Tracy CR. Utility of the RENAL nephrometry scoring system in the real world: predicting surgeon operative preference and complication risk. BJU Int. 2012;109:700-5.

11. Hayn MH, Schwaab T, Underwood W, Kim HL. RENAL nephrometry score predicts surgical outcomes of laparoscopic partial nephrectomy. BJU Int. 2011;108:876-81.

12. Ficarra V, Bhayani S, Porter J, Buffi N, Lee R, Cestari A, et al. Predictors of warm ischemia time and perioperative complications in a multicenter, international series of robotassisted partial nephrectomy. Eur Urol. 2012;61:395-402.

13. Potretzke AM, Potretzke TA, Knight BA, Vetter J, Park AM, Anderson $\mathrm{G}$, et al. Tumor diameter accurately predicts perioperative outcomes in $\mathrm{T} 1$ renal cancer treated with robotassisted partial nephrectomy. World J Urol. 2016;34:16431650.

14. Wang L, Wu Z, Ye H, Li M, Sheng J, Liu B, et al. Correlations of tumor size, RENAL, centrality index, preoperative aspects and dimensions used for anatomical, and diameter-axial-polar scoring with warm ischemia time in a single surgeon's series of robotic partial nephrectomy. Urology. 2014;83:1075-9. 
15. Patel HD, Mullins JK, Pierorazio PM, Jayram G, Cohen JE, Matlaga BR, et al. Trends in renal surgery: robotic technology is associated with increased use of partial nephrectomy. J Urol. 2013;189:1229-35.

16. Tarin T, Feifer A, Kimm S, Chen L, Sjoberg D, Coleman J, et al. Impact of a common clinical pathway on length of hospital stay in patients undergoing open and minimally invasive kidney surgery. J Urol. 2014;191:1225-30.

17. Nogueira L, Katz D, Pinochet R, Godoy G, Kurta J, Savage $\mathrm{CJ}$, et al. Critical evaluation of perioperative complications in laparoscopic partial nephrectomy. Urology. 2010;75:288-94.

18. Greene FL, Page DL, Fleming ID et al. AJCC Cancer Staging Manual, 6th ed. New York. Springer Press, 2002.

19. Dindo D, Demartines N, Clavien PA. Classification of surgical complications: a new proposal with evaluation in a cohort of 6336 patients and results of a survey. Ann Surg. 2004;240:205-13.

20. Levey AS, Stevens LA, Schmid CH, Zhang YL, lii AFC. Annals of Internal Medicine. 2015. Available at. <http://annals.org/aim>.

21. Zhang ZY, Tang Q, Li XS, Zhang Q, Mayer WA, Wu JY, et al. Clinical analysis of the PADUA and the RENAL scoring systems for renal neoplasms: a retrospective study of 245 patients undergoing laparoscopic partial nephrectomy. Int J Urol. 2014;21:40-4.

22. Becker F, Van Poppel H, Hakenberg OW, Stief C, Gill I, Guazzoni $G$, et al. Assessing the impact of ischaemia time during partial nephrectomy. Eur Urol. 2009;56:625-34.

23. Tsivian M, Ulusoy $S$, Abern $M$, Wandel $A$, Sidi AA, Tsivian A. Renal mass anatomic characteristics and perioperative outcomes of laparoscopic partial nephrectomy: a critical analysis. J Endourol. 2012;26):1307-13.

24. Zheng Y, Espiritu P, Hakky T, Jutras K, Spiess PE. Predicting ease of perinephric fat dissection at time of open partial nephrectomy using preoperative fat density characteristics. BJU Int. 2014;114:872-80.
25. Song C, Bang JK, Park HK, Ahn H. Factors influencing renal function reduction after partial nephrectomy. J Urol. 2009;181:48-53;discussion 53-4.

26. Simhan J, Smaldone MC, Tsai KJ, Canter DJ, Li T, Kutikov $A$, et al. Objective measures of renal mass anatomic complexity predict rates of major complications following partial nephrectomy. Eur Urol. 2011;60:724-30.

27. Yossepowitch 0, Eggener SE, Serio A, Huang WC, Snyder ME, Vickers AJ, et al. Temporary renal ischemia during nephron sparing surgery is associated with short-term but not long-term impairment in renal function. J Urol. 2006;176:1339-43.

28. Turna B, Frota R, Kamoi K, Lin YC, Aron M, Desai MM, et al. Risk factor analysis of postoperative complications in laparoscopic partial nephrectomy. J Urol. 2008;179:128994.

29. Mayer WA, Godoy G, Choi JM, Goh AC, Bian SX, Link RE. Higher RENAL Nephrometry Score is predictive of longer warm ischemia time and collecting system entry during laparoscopic and robotic-assisted partial nephrectomy. Urology. 2012;79:1052-6.
Correspondence address:

Jonathan A. Coleman, MD Department of Surgery Urology Service, Memorial Sloan Kettering Cancer Center New York, USA 1275 York Ave New York, 10065, USA Telephone: + 1646 422-4432 E-mail: colemanj@mskcc.org 